\title{
Stability of Nonlinear Te Surface Waves along the Boundary of Left-Handed Material
}

\author{
Hana Mohammed Mousa \\ Physics Department, Al Azhar University, Gaza, Palestinian Authority \\ Email: H.Mousa@alazhar-gaza.edu
}

Received March 30, 2012; revised April 28, 2012; accepted May 7, 2012

\begin{abstract}
This paper is concerned with the stability characteristics of nonlinear surface waves propagating along a left-handed substrate (LHM) and a non-linear dielectric cover. These characteristics have been simulated numerically by using the perturbation method. The growth rate of perturbation is computed by solving the dispersion equation of perturbation. I found that the stability of nonlinear surface waves is affected by the frequency dependence of the electric permittivity $\varepsilon_{h}$ and magnetic permeability $\mu_{h}$ of the LHM. The spatial evolution of the steady state field amplitude is determined by using computer simulation method. The calculations show that with increasing the effective refractive index $n_{x}$ at fixed saturation parameter $\mu_{p}$, the field distribution is sharpened and concentrated in the nonlinear medium. The waves are stable of forward and backward behavior. At higher values of $n_{x}$, attenuated backward waves are observed.
\end{abstract}

Keywords: Nonlinear Waves; Wave-Guides; Dispersion Relation; Left-Handed Material; Growth Rate; Stability

\section{Introduction}

Recently, there has been great interest in new type of electromagnetic materials called left-handed media [1]. Over fifty years ago, Veselago was the first to consider the left-handed meta-material (LHM) which he defined as media with simultaneously negative and almost real electric permittivity and magnetic permeability in some frequency range [2]. The electric and magnetic fields form a left-handed set of vectors with the wave vector [3]. These materials have been shown to exhibit unique properties, such as Snell law and Doppler shift. Smith, et al. [4] have built these materials by using two dimensional arrays of splitting resonators and wires and are operating the microwave range. Nonlinear surface waves propagating along the interface of linear and nonlinear media have a number of novel extraordinary properties which attracted attention of many investigators [5-8]. Understanding the stability of nonlinear surface waves is essential for the exploitation of these waves in various devices. There are numbers of approaches to the problem both using numerical simulations methods by Akhmediev et al. [8] and Moloney et al. [5] and analytical methods by Tran [6] which has been based on steady-state solutions to a nonlinear wave equation which contains an intensity dependent refractive index. The question is whether these wave solutions are stable on propagation of waves. Akhmediev et al. [8] had shown when the growth rate of perturbation of waves $\delta$ is real, the sur- face waves are unstable and when $\delta$ is imaginary, the waves are stable. Akhmediev et al. [7] explained the stability behavior of antisymmetric and symmetric solutions of a linear core sandwiched between two nonlinear media. They showed that the antisymmetric wave is stable at high values of the propagation constant, in contrast to the symmetric wave. Hasegawa [9] studied the soliton effects in various fibers, he reported that, optical soliton is formed by a balance between the dispersion velocity of the waves and the Kerr nonlinearity of the fiber. Sukhorukov et al. investigated the Spatial optical solitons in waveguide arrays, they predicted, two-dimensional (2D) networks of nonlinear waveguides which allow a possibility of realizing useful functional operations with discrete solitons such as signal switching, blocking, routing, and time gating [10,11]. Setzpfandt et al. described discrete solitons in quadratic waveguide arrays [12]. Their results demonstrated that a power threshold may appear for soliton formation, leading to a suppression of beam self-focusing which explains recent experimental observations. Shabat and Mousa have studied the stability of nonlinear surface waves along the boundary of linear semiconductor [13] and along the boundary of lateral antiferromagnetic/nonmagnetic superlattice (LANS) [14]. These studies were carried out in a media with positive refractive index. Such media are called right handed materials.

This paper is concerned with the stability of nonlinear surface waves propagating along the boundary of left- 
handed media [1] (LHM).

To study the stability of the corresponding surface waves, it is necessary to select a particular form of the frequency dependence of the electric permittivity $\varepsilon_{h}$ and magnetic permeability $\mu_{h}$ of the LHM, I solve this problem by using computer simulation method [15].

The geometry is shown in Figure 1. It consists of a non-linear semi-infinite cladding contact everywhere to a linear, semi-infinite LHM substrate at $y=0$ planar interface. The coordinate system is such that, the $y$ axis is normal to the interface and the wave vector is directed along the $x$ axis.

\section{Theoretical Analysis}

Since the wave propagation is in $x$-direction then, the Maxwell equations for $S$-polarized wave (TE) are reduced to the following Equation [8]

$$
\nabla^{2} E+\varepsilon\left(y,|E|^{2}\right) E=0
$$

The dielectric constant of the linear medium in the region $y<0$ is $\varepsilon_{h}$, while the dielectric function in region $y>0$ is:

$$
\varepsilon^{\mu l}=\varepsilon_{3}+\alpha|E|^{2}
$$

Assuming that the nonlinear medium is self-focusing, the solution of the wave equation which is polarized along the $z$-axis is:

$$
E_{z}(x, y)=\alpha^{1 / 2} A(x, y) e^{i\left(n_{x} x-\omega t\right)}
$$

where $A(x, y)$ is a slowly varying field envelope, $n_{x}$ is the effective refractive index.

By substituting Equation (3) into Equation (1), the equation for the slowly varying amplitude $A(x, y)$ is then [5]

$$
2 i n_{x} \frac{\partial A}{\partial x}+\frac{\partial^{2} A}{\partial y^{2}}-k_{2}^{2}(y) A+\frac{\alpha}{\alpha_{0}}|A|^{2} A=0
$$

where

$$
k_{2}^{2}(y)=n_{x}^{2}-\varepsilon_{3}
$$

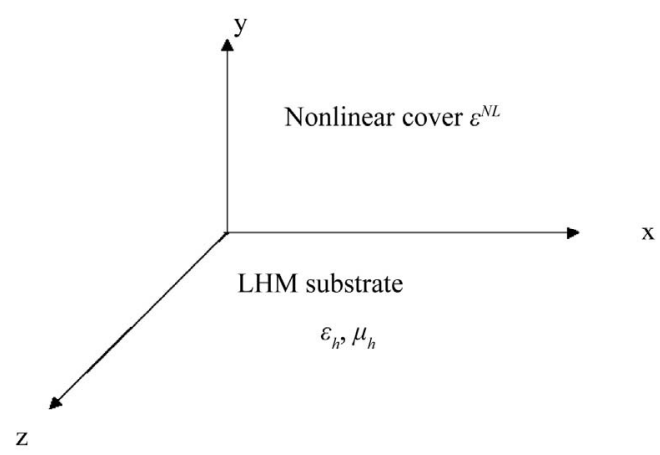

Figure 1. Configuration of a single interface nonlinear cover/LHM substrate structure. is the decay constant of the nonlinear medium, $\varepsilon_{3}$ is the linear part dielectric function of the non linear medium, the coordinates $x$ and $y$ are normalized by the factor $\omega / c$, and the fields are normalized by the factor $\alpha_{0}^{1 / 2}$, where $\omega$ is the wave angular frequency, $c$ is the light velocity in free space, and $\alpha_{0}$ is the non-linearity coefficient.

The investigation of the stability of nonlinear surface wave (NSW) propagation along the interface between the linear and non linear medium has been focused in looking for the steady-state solution $A(x, y)=A_{0}(y)$ of Equation (4a) in the proposed structure as:

$A_{0}(y)=$

$\left\{\begin{array}{l}2^{\frac{1}{2}}\left(k_{2}^{2}-k_{1}^{2}\right)^{1 / 2} e^{k_{1} y}, y<0, \text { for linear medium } \\ 2^{\frac{1}{2}} k_{2} \sec h\left(k_{2}\left(y-y_{0}\right)\right), y>0 \text {, for nonlinear medium }\end{array}\right.$

At the interface between the two media $y=0$, we assume the condition that the dielectric constant of the linear medium $\varepsilon_{h}>\varepsilon_{3}$ and

$$
y_{0}=\frac{1}{2 k_{2}} \ln \frac{k_{2}+k_{1}}{k_{2}-k_{1}}
$$

where

$$
k_{1}^{2}(y)=n_{x}^{2}-\varepsilon_{h} \mu_{h}
$$

is the decay constant of the linear medium.

Both a negative dielectric permittivity and permeability are written as [3]:

$$
\varepsilon_{h}(\omega)=1-\frac{\omega_{p}^{2}}{\omega^{2}}, \mu_{h}(\omega)=1-\frac{F \omega^{2}}{\omega^{2}-\omega_{0}^{2}}
$$

with plasma frequency $\omega_{p}$ and resonance frequency $\omega_{0}$.

To determine the stability criterion for NSWs, I numerically stimulated the steady-state solution of Equation (4a) with small perturbation as [8]:

$$
A(x, y)=A_{0}(y)+\mu_{p} f(x, y)
$$

where $f(x, y)$ is a perturbation function of the steadystate solution, $\mu_{p}$ is the saturation parameter.

Substituting Equation (7) into Equation (4a), we can obtain:

$$
\begin{aligned}
& 2 i n_{x} \frac{\partial f}{\partial x}+\frac{\partial^{2} f}{\partial y^{2}}-k_{2}^{2}(y) f \\
& +\frac{\alpha}{\alpha_{0}} A_{0}^{2}(y)\left(2 f+f^{*}\right)=0
\end{aligned}
$$

We shall consider the $z$ dependence of the perturbation function, so that the function can be written in the form [8]: 


$$
f(x, y, z)=\frac{1}{2}\left[(u+v) e^{(\delta x+i r z)}+\left(u^{*}-v^{*}\right) e^{\left(\delta^{*} x-i r z\right)}\right]
$$

where $u$ and $v$ are functions of $y$ only. We take the case $r^{2}=\delta^{2}$ for nonlinear medium.

Substituting Equation (9) into Equation (8), we obtain the set of differential equations which have solutions decay as $\bar{y} \rightarrow \infty$ for self focused waves in nonlinear medium of the form:

$$
\begin{aligned}
& u=c_{1} e^{-p \bar{y}}\left[-i \xi+2 p \tanh \bar{y}+2 \tanh ^{2} \bar{y}\right] \\
& +c_{2} e^{-p^{*} \bar{y}}\left[+i \xi+2 p^{*} \tanh \bar{y}+2 \tanh ^{2} \bar{y}\right] \\
& v=c_{1} e^{-p \bar{y}}[2+i \xi-2 p \tanh \bar{y}] \\
& -c_{2} e^{-p^{*} \bar{y}}\left[2-i \xi-2 p^{*} \tanh \bar{y}\right]
\end{aligned}
$$

where

$$
\bar{y}=k_{2}\left(y-y_{0}\right), \quad \xi=\xi^{\prime} / k_{2}^{2}, \quad \xi^{\prime}=2 n_{x} \delta,
$$

where $p=(1+i \xi)^{1 / 2}, \quad c_{1}, c_{2}$ are constants to be determined from the boundary condition, and primes denote the derivatives with respect to $\bar{y}$.

In a linear medium, the solutions are decaying as $\bar{y} \rightarrow-\infty$,

$$
u=A_{1} e^{s \bar{y}}+A_{2} e^{s^{*} \bar{y}}, \quad v=A_{1} e^{s \bar{y}}-A_{2} e^{s^{*} \bar{y}}
$$

where $s=\left(\frac{k_{1}^{2}}{k_{2}^{2}}-i \xi\right)^{1 / 2}$, and $A_{1}, A_{2}$ are constants to be determined from the boundary conditions. For a surface wave $\xi$ is either real or imaginary, thus by a bit of algebra we can obtain a dispersion relation for determining $\xi$ of the form [8]:

$$
\begin{aligned}
& \left|p(1+i \xi)-2 i \xi t-3 p t^{2}+2 t^{3}-s(p-t)^{2}\right|^{2} \\
& -\left(1-t^{2}\right)^{2}|p-2 t+\bar{s}|^{2}=0
\end{aligned}
$$

where $t=\tanh \left(k_{2} y_{0}\right)$ which implies $0<t<1$ and

$$
\tanh \left(k_{0} k_{z} y_{0}\right)=\frac{k_{1}}{k_{2}}
$$

Equation (12a) may be solved analytically by expanding each of the two expressions under the absolute value in terms of $\xi$ up to the fourth order and by calculating the absolute values of these expressions, one obtains that [8]

$$
\xi_{r}^{2}=0.533(1-2 t)
$$

when $t<1 / 2, \quad \xi_{r}^{2}>0 \Rightarrow \xi_{r}$ is real, the growth rate $\delta$ is related to $\xi_{r}$ by Reference [8], $\delta=\xi_{r} k_{2}^{2} / 2 n_{x}$ which causes the NSW to be unstable.

When $t>1 / 2, \quad \xi_{r}^{2}<0 \Rightarrow \xi_{r}$ is imaginary where $\delta$ becomes imaginary and NSW is stable. At $t=1 / 2, n_{x}$ is the critical refractive index in this case.

The evolution of the perturbed field amplitude $A(y)$ at the propagation distance $x$ is calculated by the determination of the constants $c_{1}, c_{2}, A_{1}, A_{2}$ through application of the boundary conditions at $\mathrm{y}=0$ as [5]:

$$
\text { (1) } E_{z_{l}}=E_{z_{N l}}
$$

It is found by substituting Equations (5) \& (7) into Equation (3), which results in

$$
\text { (2) } \frac{\partial E_{z n l}}{\partial y}=\frac{\partial E_{z l}}{\partial y}
$$

Since the wave function $u$ vanishes at the boundary, say $y=10$ then (3) $u_{N_{l}}=0$ at $y=10$

$$
\text { (4) } u_{l}=0 \text { at } y=-10
$$

At the initial perturbation where $x=0$, it is convenient to take $c_{2}=c_{1}^{*}$ and $A_{2}=A_{1}^{*}$, then by solving the two Equations (14) and (15), we can obtain the values of the constants $c_{1}, c_{2}, A_{1}, A_{2}$. By numerical simulation method it is easy to study the evolution of the steady-state field amplitude

$$
A(x, y, z) \text {, at } x=0, x=2.9 \text {, and } x=3 \text {. }
$$

The variation of the energy integral of the nonlinear surface waves with $n_{x}$ is also calculated analytically for different values of the wave frequency through the integral of square perturbed field amplitude in linear and nonlinear medium as [8]

$$
I=\int_{-\infty}^{0}\left|A_{l}(x, y)\right|^{2} \mathrm{~d} y+\int_{0}^{\infty}\left|A_{N L}(x, y)\right|^{2} \mathrm{~d} y
$$

where $A_{l}(x, y), A_{N L}(x, y)$ are the perturbed field amplitude in linear and nonlinear medium respectively.

\section{Computer Simulation and Discussion}

Some numerical calculations are presented for the simulation of the stability Equation (7) of the proposed structure, which consists of LHM substrate and a nonlinear dielectric cover. Computer simulation software (Maple) [15] is used in our computation, where the run takes a reasonable usage time. The parameters are [3] as follows: $\omega_{p} / 2 \pi=10 G H z, \omega_{0} / 2 \pi=4 G H z$, and $F=0.56$ and for the non-linear medium, $\varepsilon_{3}=2.25$. Figures 2(a)-(c) show that for this set of parameters, the frequency range in which both $\varepsilon_{h}$ and $\mu_{h}$ are negative is from $4 \mathrm{GHz}$ to $6 \mathrm{GHz}$.

For increasing values of wave frequency $(\omega / 2 \pi)$, Figures 2(a)-(c) display the spatial evolution of steady state field amplitude $A_{l}(x, y), A_{N L}(x, y)$ as a function of the wave frequency $(\omega / 2 \pi)$. I found that at $n_{x}=4$ and wave frequency $(\omega / 2 \pi=4.3 \mathrm{GHz}),\left(\varepsilon_{h}, \mu_{h}\right)$ are of values $(-4.4,-3.185)$ respectively as computed from Equation (6c). The perturbed waves are unstable where the growth 


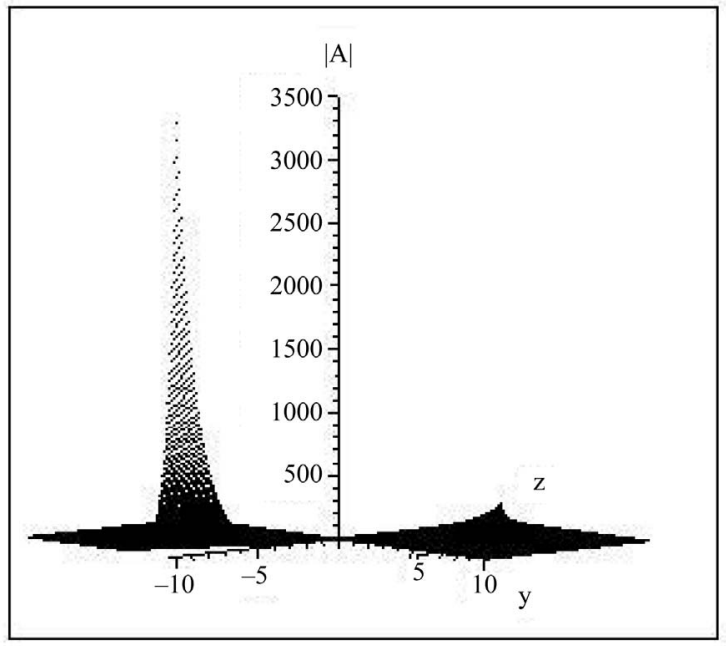

(a)

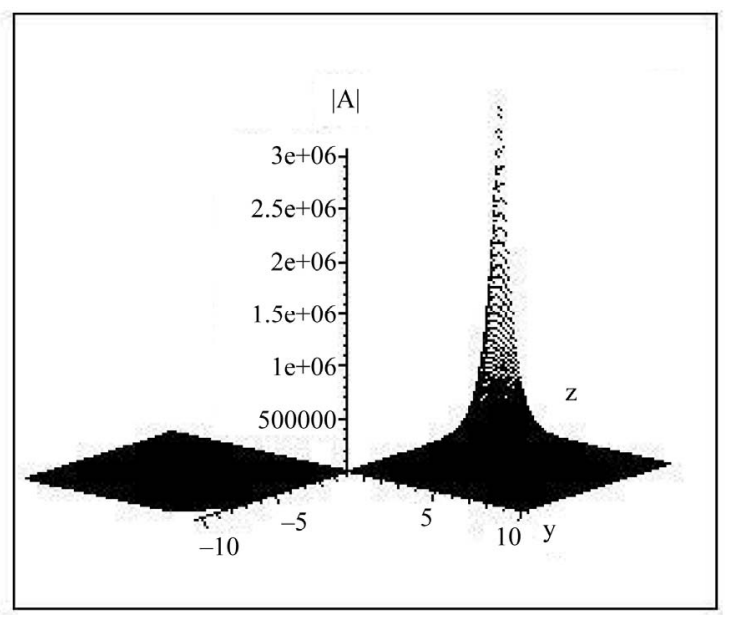

(b)

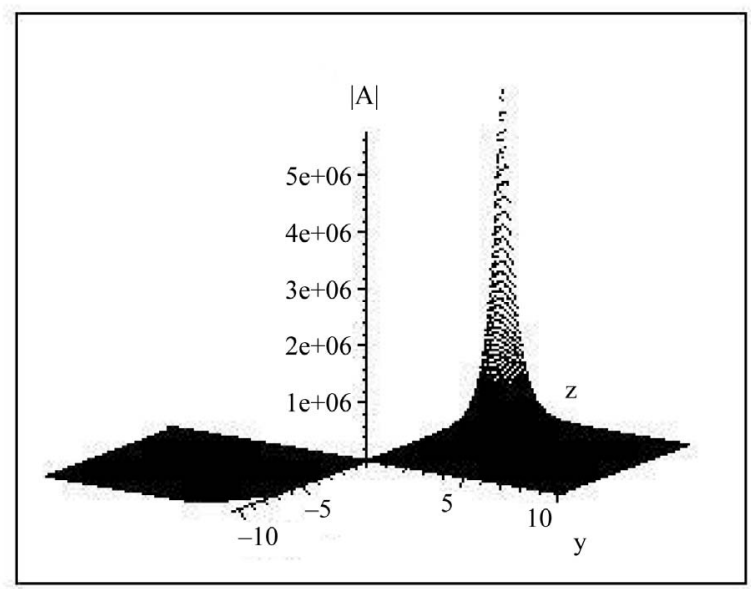

(c)

Figure 2. The field distribution of the nonlinear surface waves $\mathrm{A}(\mathrm{y}, \mathrm{z})$ for (a) $\omega / 2 \pi=4.3 \mathrm{GHz}$, growth rate $\delta=0.626$; (b) $(\omega / 2 \pi)=5.6 \mathrm{GHz}, \delta=1.337 * \mathrm{I} \&$ (c) $\omega / 2 \pi=5.9 \mathrm{GHz}, \delta=$ $1.347 *$ I for $\mu_{p}=0.3, \omega_{p} / 2 \pi=10 \mathrm{GHz}, \omega_{0} / 2 \pi=4 \mathrm{GHz}, \varepsilon_{3}=$ $2.25 \&$ propagation distance $x=3$. rate of perturbation $\delta$ is real $(\delta=0.626)$. The decay constant of NSW in nonlinear medium $k_{2}(y)=3.708$ and the decay constant of NSW in linear medium $k_{1}(y)$ $=1.39, t=0.3755$ as computed from Equations $(4 \mathrm{~b}),(6 \mathrm{~b})$ $\&(12 b)$, respectively. For increasing values of $(\omega / 2 \pi)$ to $(5.6 \mathrm{GHz}$ and $5.9 \mathrm{GHz})$ the $\varepsilon_{h}$ changes to the values ($2.19,-1.875)$ while $\mu_{h}$ changes to the values $(-0.144,-$ $0.037)$, the $k_{1}(y)$ is increased to $(3.96,3.99)$ and $k_{2}(y)$ is constant because $n_{x}$ is constant, $\mathrm{t}$ is increased to $(1.0679,1.076)$ so, the growth rate $\delta$ becomes imaginary of values $(1.337 * \mathrm{I}, 1.347 * \mathrm{I})$ respectively. The field distribution is sharpened where the wave's turns from unstable to stable waves and concentrated in the non linear medium. This means that the stability of the waves is affected with the wave frequency.

Figures 3(a)-(c) display the spatial evolution of steady state field amplitude $A_{l}(x, y), A_{N L}(x, y)$ as a function of the refractive index $n_{x}$. I found that at wave frequency $(\omega / 2 \pi=4.9 \mathrm{GHz}), \varepsilon_{h}$ is of value $(-3.169) \& \mu_{h}$ is of value $(-0.682)$. At $n_{x}=3$, the perturbed waves are stable where the growth rate of perturbation $\delta$ is imaginary $\left(\delta=0.8266^{*} \mathrm{I}\right)$. The decay constant of NSW in nonlinear medium $k_{2}(y)=2.598$ and the decay constant of NSW in linear medium $k_{1}(y)=2.615, t=1.006$. For increasing value of $n_{x}$ to $(4.5)$ the $k_{2}(y)$ is increased to (4.253) \& $k_{1}(y)$ is increased to (4.242) and $t$ is decreased to (1.002) so, the growth rate $\delta$ still imaginary of value $(1.463 * \mathrm{I})$ respectively where the waves shifted to the nonlinear medium, with the subsequent excitation of the nonlinear stable surface waves of high energy (soliton). At $n_{x}=5$, the perturbed waves still stable of decreasing energy, the growth rate of perturbation ( $\delta=$ $1.664 * \mathrm{I})$. The decay constant of NSW in nonlinear medium $k_{2}(y)=4.778$ and the decay constant of NSW in linear medium $k_{1}(y)=4.769, \mathrm{t}=1.0019$. Figure 4, illustrates the energy flow $I$ of the nonlinear surface waves as a function of $n_{x}$ for various values of $\mu_{p}$. For $\mu_{p}=0.1$, the wave's energy is increased by increasing $n_{x}$ where the waves are forward traveling. For increasing value of $\mu_{p}$ to $(0.3)$, the high wave energy is concentrated at $n_{x}=4.5$ of forward traveling \& then decreases by increasing $n_{x}$. It shows that at values of $n_{x} \succ 6$, the energy becomes negative, where the waves can be switched to the backward propagation as an effect of the LHM.

These results are different from that obtained for the magnetic medium such as lateral antiferromagnetic/nonmagnetic superlattice (LANS) [14] and gyrodielectric medium as a semiconductor [13]. The existence of the magnetic matter causes the growth rate to be always real and the waves are always unstable. For a semiconductor substrate, the waves are stable of forward traveling.

\section{Conclusions}

The stability characteristics of nonlinear surface waves 


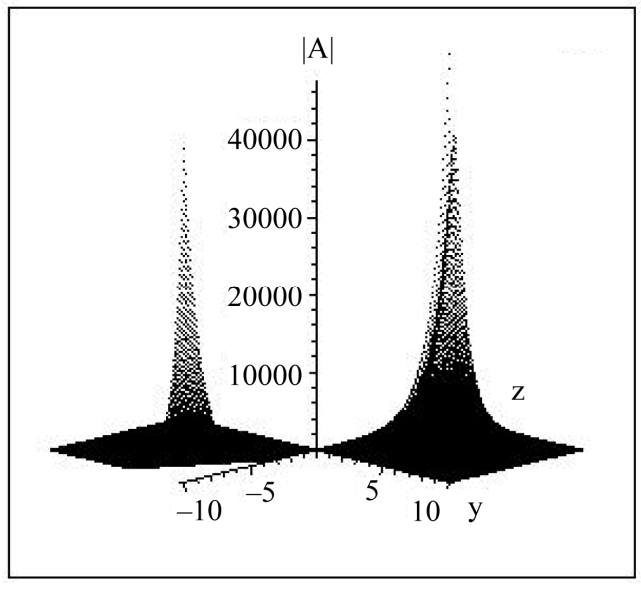

(a)

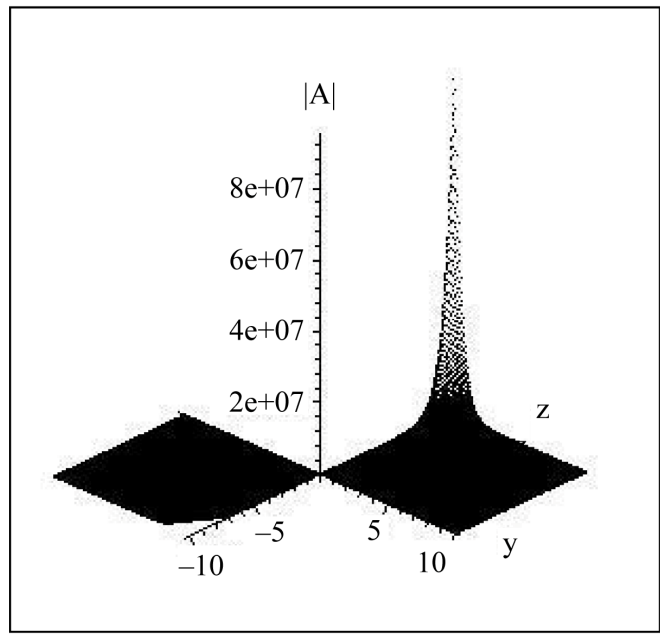

(b)

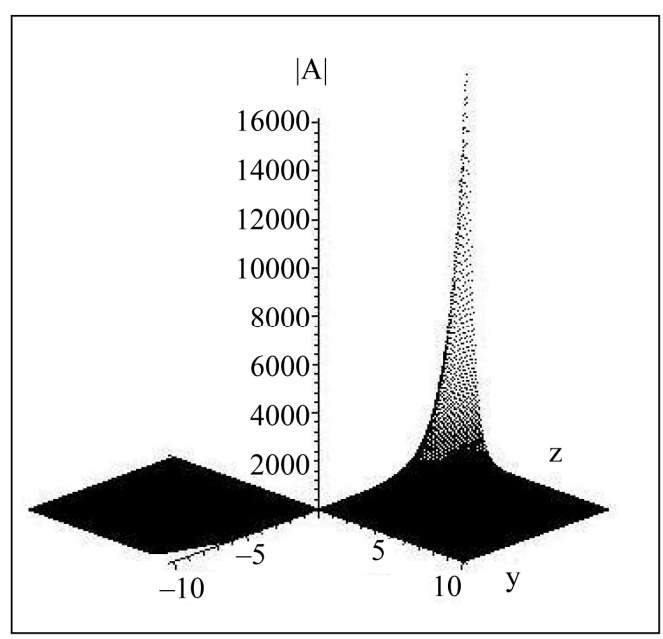

(c)

Figure 3. The field distribution of the nonlinear surface waves $\mathrm{A}(\mathrm{y}, \mathrm{z})$ for (a) $n_{x}=3$, growth rate $\delta=0.8266$; (b) $n_{x}=$ $4.5, \delta=1.463 * I$ and (c) $n_{x}=5, \delta=1.664 * I$ for $\mu_{p}=0.3, \omega_{p} / 2 \pi$ $=10 \mathrm{GHz}, \omega / 2 \pi=4.9 \mathrm{GHz}, \omega_{0} / 2 \pi=4 \mathrm{GHz}, \varepsilon_{3}=2.25$ and propagation distance $x=3$.

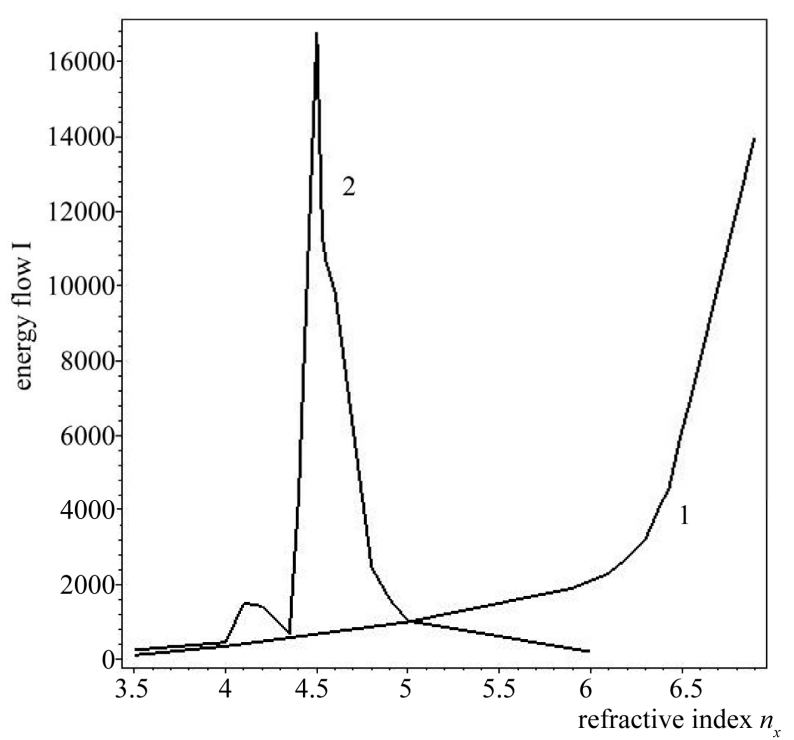

Figure 4. The energy flow $I$ of the nonlinear surface waves as a function of $n_{x}$ for (1) $\mu_{p}=0.1$ and (2) $\mu_{p}=0.3, \omega_{p} / 2 \pi=$ $10 \mathrm{GHz}, \omega / 2 \pi=4.9 \mathrm{GHz}, \omega_{0} / 2 \pi=4 \mathrm{GHz}, \varepsilon_{3}=2.25$ and propagation distance $x=3$.

propagating along a left-handed substrate(LHM) and a non-linear dielectric cover are investigated. I found that, the stability of the waves in LHM can be controlled by the frequency dependence of the electric permittivity and magnetic permeability of the LHM. By increasing the effective refractive index at fixed saturation parameter, the field distribution is sharpened which is implying the possibility of optical switching and the field concentrated in the nonlinear medium (optical soliton) which is useful for practical ultrahigh-speed communications. At higher values of $n_{x}$, attenuated backward waves are observed. I believe that the stability which has been investigated and reported here may provide new opportunities for the design of future microwave-photonic devices.

\section{REFERENCES}

[1] L. Hu and S. T. Chui, "Characteristics of Electromagnetic Wave Propagation in Uniaxially Anisotropic Left-Handed Materials," Physical Review: B, Vol. 66, No. 8, 2002, pp. 085108-085115. doi:10.1103/PhysRevB.66.085108

[2] V. G. Veselago, "The Electrodynamics of Substances with Simultaneously Negative Values of Permittivity and Permeability," Soviet Physics Uspekhi, Vol. 10, No. 4, 1967, p. 509. doi:10.1070/PU1968v010n04ABEH003699

[3] I. V. Shadrivov, A. A. Sukhorakov and Y. S. Kivshar, "Nonlinear Surface Waves in Left-Handed Material," Physical Review: E, Vol. 69, No. 1, 2004, p. 016617. doi:10.1103/PhysRevE.69.016617

[4] D. R. Smith, W. J. Padilla, D. C. Vier, S. C. Nemat-Nasser and S. Schultz, "Composite Medium with Simultaneously Negative permeability and Permittivity," Physical Review Letters, Vol. 84, No. 18, 2000, p. 4184. 


\section{doi:10.1103/PhysRevLett.84.4184}

[5] J. V. Moloney, J. Ariyasu and G. I. Stegeman, "Stability of Nonlinear Stationary Waves Guided by a Thin Film Bounded by Nonlinear Media," Applied Physics Letter, Vol. 48, No. 13, 1986, p. 826. doi:10.1063/1.96680

[6] H. T. Tran, "Stability of Stationary Dark Waves Guided by Nonlinear Surfaces and Waveguides," Journal of the Optical Society of America B, Vol. 11, No. 5, 1994, pp. 789-797. doi:10.1364/JOSAB.11.000789

[7] N. Akhmediev, A. Ankiewicz and H.-T. Tan, "Stability Analysis of Even and Odd Waves of Symmetric Nonlinear Planar Optical Waveguides," Journal of the Optical Society of America B, Vol. 10, No. 1, 1993, pp. 230-236. doi:10.1364/JOSAB.10.000230

[8] N. N. Akhmediev, "Nonlinear Surface Electromagnetic Phenomena," In: H. E. Ponath and G. I. Stegeman, Eds., The Problem of Stability and Excitation of Nonlinear Surface Waves, Elsevier, Horth Holland, 1991, p. 289.

[9] A. Hasegawa, "Soliton Effects in Optical Waveguides," Reports on Progress in Physics, Vol. 65, No. 6, 2002, pp. 999-1024. doi:10.1088/0034-4885/65/6/203

[10] A. A. Sukhorukov, Y. S. Kivshar, H. S. Eisenberg and Y. Silberberg, "Spatial Optical Solitons in Waveguide Ar- rays," IEEE Journal of Quantum Electron, Vol. 39, No. 1, 2003, pp. 31-50. doi:10.1109/JQE.2002.806184

[11] M. Johansson, A. A. Sukhorukov and Y. S. Kivshar, "Discrete Reduced-Symmetry Solitons and Second Band Vortices in Two Dimensional Nonlinear Waveguide Arrays," Physical Review: E, Vol. 80, No. 4, 2009, pp. 046604046619. doi:10.1103/PhysRevE.80.046604

[12] F. Setzpfandt, A. A. Sukhorukov and T. Pertsch, "Discrete Quadratic Solitons with Competing Second-Harmonic Components," Physical Review A, Vol. 84, No. 5, 2011, pp. 053843-053851. doi:10.1103/PhysRevA.84.053843

[13] H. M. Mousa and M. M. Shabat, "Stability of Nonlinear Te Surface Waves along the Boundary of Linear Gyrodielectric Media," International Journal of Modern Physics $B$, Vol. 21, No. 26, 2007, pp. 4487-4493. doi:10.1142/S0217979207038009

[14] M. M. Shabat and H. M. Mousa, "Stability of Nonlinear Te Surface Waves along the Interface of Nonlinear Dielectric and Superlattices Media," The Islamic University Journal, Vol. 14, No. 1, 2006, pp. 135-145.

[15] “Maple Software," Version 9, 615 Kumpf Drive, Waterloo, 2004. 